\title{
Research Paper \\ Effect of Anesthetics on Oxidant and Antioxidant Parameters After Inguinal Hernia Surgery in Older Patients
}

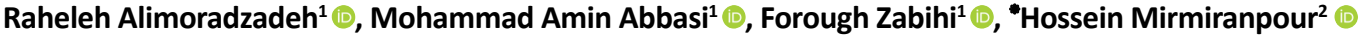 \\ 1. Clinical Research Development Unit, Firoozabadi Hospital, Iran University of Medical Sciences, Tehran, Iran. \\ 2. Endocrinology and Metabolism Research Center, Vali-Asr Hospital, Tehran University of Medical of Sciences, Tehran, Iran.
}

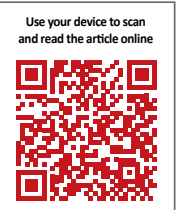

dtation: Alimoradzadeh R, Abbasi MA, Zabihi F, Mirmiranpour H. [Effect of Anesthetics on Oxidant and Antioxidant Parameters after Inguinal Hernia Surgery in Older Patients (Persian)]. Iranian Journal of Ageing. 2021; 15(4):524-533. https://doi. org/10.32598/sija.15.4.3007.1

https://doi.org/10.32598/sija.15.4.3007.1

Keywords:

Anesthesia, Inguinal hernia, Isoflurane, Lidocaine, Oxidant, Antioxidant

\section{A B S T R A C T}

Objectives The effects of anesthesia techniques and anesthetics on different systems and organs of the body, especially the immune system, in older patients undergoing surgery, have always been of interest to researchers. This study aims to compare the effects of general anesthesia with isoflurane and spinal anesthesia with lidocaine on oxidative and antioxidant parameters in older patients with inguinal hernia surgery.

Methods \& Materials This is a double-blinded randomized clinical trial with parallel design conducted on 70 older patients referred to the surgery department of hospitals in Tehran, Iran during 2018-2019. They were randomly divided into two groups of 35; the first group received general anesthesia with isoflurane and the second group received spinal anesthesia with lidocaine. Blood sampling was performed in two stages; one day before and one day after surgery. Advanced Glycation End Products (AGEs), Advanced Oxidation Protein Products (AOPP), Malondialdehyde (MDA), oxidized Low-Density Lipoprotein (LDL), Ferric-Reducing Ability of Plasma (FRAP), glutathione peroxidase, superoxide dismutase and catalase levels were measured before and after anesthesia using standard methods. Collected data were analyzed in SPSS v.22 software.

Results The Mean \pm SD age of patients in the lidocaine group was $69.94 \pm 8.31$ years and in the isoflurane as $70.23 \pm 4.98(P>0.81)$. In the isoflurane group, there was a significant difference between pre- and postoperative levels of AOPP, MDA, oxidized LDL, FRAP, catalase, glutathione peroxidase and superoxide dismutase. In the lidocaine group, this difference was significant in MDA, oxidized LDL, catalase and superoxide dismutase.

Conclusion Given the positive effects of isoflurane on oxidative and antioxidant parameters in older patients with inguinal hernia surgery, it is recommended that this anesthetic be considered in the selection of anesthesia methods and drugs for this group of older patients.

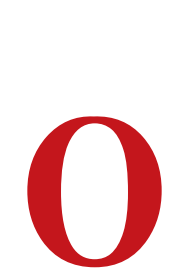

\section{Extended Abstract}

\section{Introduction}

xidant and antioxidant levels have a fundamental relationship with the elderly's immune system and their degree of exposure to anesthetics in surgery is important [3]. Isoflurane, by affecting hydrogen peroxide (as an oxidant), changes its amount in serum [4]. This anesthetic can also affect antioxidant properties and increase DNA damage [5]. Lidocaine is used as an anesthetic for spinal anesthesia [6]. Spinal anesthesia with

\section{* Corresponding Author:}

Hossein Mirmiranpour, PhD.

Address: Endocrinology and Metabolism Research Center, Vali-Asr Hospital, Tehran University of Medical of Sciences, Tehran, Iran.

Tel: +98 (912) 3388654

E-mail: h_mirmiranpoor@yahoo.com 
lidocaine further modulates oxidative stress and the amount of antioxidants to the normal range compared to general anesthesia [7]. Study of changes in oxidant and antioxidant parameters after anesthesia with isoflurane and spinal anesthesia with lidocaine, helps to choose a safer method for the elderly with underlying diseases $[1,2]$.

\section{Methods \& Materials}

This double-blinded clinical trial study (ethics code: IR.IUMS.FMD.REC.1397.304) was performed on older patients aged 60-70 years who were not using oxidant or antioxidant drugs and had no acute cardiovascular disease, acute renal failure, liver cirrhosis, malignancy and infection. Patients were randomly divided into two groups of 35 . In one group, anesthesia with isoflurane and in the other group, spinal anesthesia with lidocaine was used. Sample collection was done in two stages; one day before and one day after inguinal hernia surgery. Samples obtained at baseline were considered as control group and those obtained after surgery were considered as case group. AGEs, Advanced Oxidation Protein Products (AOPP), Malondialdehyde (MDA), oxidized LDL, Ferric-Reducing Ability of Plasma (FRAP), glutathione peroxidase, superoxide dismutase and catalase were measured as immune system indices by standard methods before and after anesthesia. Enzymatic colorimetric method was used to measure the antioxidant activity of catalase, glutathione peroxidase and superoxide dismutase and the oxidant activity of MDA. The oxidized LDL was measured by ELISA technique. Determination of AOPP was performed using a spectrophotometric method described by Kalousová et al. [??].In measuring the antioxidant capacity of plasma or FRAP, 750 $\mu \mathrm{l}$ of reagent was added to $25 \mu \mathrm{l}$ of plasma in the test tubes and their absorbance at $593 \mathrm{~nm}$ was read using a spectrophotometer. For statistical analysis, SPSS software V. 20 was used and quantitative data were described using mean and standard deviation.

\section{Results}

In the present study, 70 older patients (34 males) with a history of inguinal hernia surgery, were studied. Their mean age in the two groups $(69.94 \pm 5.15$ vs. $70.23 \pm 4.98$ years; $P$ $=0.81$ ), and their other demographic characteristics were not significantly different from each other. The mean systolic pressure $(124.29 \pm 9.16$ vs. $123.7 \pm 9.10 \mathrm{~mm} \mathrm{Hg}$; $\mathrm{P}=$ $0.79)$ and diastolic pressure (72.29 \pm 6.89 vs. $72.29 \pm 7.31$ $\mathrm{mmHg} ; \mathrm{P}=0.99$ ) measured before the surgery was not significantly different between the two groups. After surgery, although the measured systolic pressure in the spinal anesthesia group was higher than in the general anesthesia group, but this difference was not significant (127.43 \pm 8.52 vs. $126.29 \pm 8.43 \mathrm{mmHg} ; \mathrm{P}=0.58)$. The same results was reported for their diastolic pressure after surgery $(76 \pm 5.53$ vs. $75.71 \pm 6.08 \mathrm{mmHg} ; \mathrm{P}=0.83$ ).

In statistical analysis of oxidative and antioxidant indices, results showed a significant difference between pre- and postoperative status in AOPP, MDA, oxidized LDL, FRAP, catalase, glutathione peroxidase and superoxide dismutase in the group received general anesthesia with isoflurane. In the spinal anesthesia group, there was a significant difference between pre- and postoperative status in MDA, oxidized LDL, catalase, and superoxide dismutase.

\section{Conclusion}

The pre- and postoperative levels of AOPP, MDA, oxidized LDL, FRAP, catalase, glutathione peroxidase and superoxide dismutase was significantly different in the patients with inguinal hernia surgery received general anesthesia with isoflurane. In the patients received spinal anesthesia with lidocaine, the difference was significant only in MDA, oxidized LDL, catalase, and superoxide dismutase levels. Considering the observed effects of anesthetics on oxidative and antioxidant indices in elderly patients, especially in those receiving anesthesia with isoflurane, these results should be considered in the selection of anesthesia methods and gases for the elderly undergoing inguinal hernia surgery. It is recommended to conduct further studies on the elderly patients using a larger sample size so that the results can be generalized with more confidence.

\section{Ethical Considerations}

\section{Compliance with ethical guidelines}

This study was approved by the Research Ethics Committee of Iran University of Medical Sciences (Code: IR.IUMS.FMD.REC.1397.304). All ethical principles are considered in this article. The participants were informed about the purpose of the research and its implementation stages. They were also assured about the confidentiality of their information and were free to leave the study whenever they wished, and if desired, the research results would be available to them.

\section{Funding}

This research did not receive any grant from funding agencies in the public, commercial, or non-profit sectors. 


\section{Authors' contributions}

Conceptualization, research: Hossein Mirmiranpour and Raheleh Alimoradzadeh; Editing and final approval: All authors.

\section{Conflicts of interest}

The authors declare no conflict of interest. 


\title{
اثرات تكنيكهاى بىهوشى بر شاخصهاى أنتى اكسيدان و اكسيدان بعد از جراحى هرنى اينكَوينال در بيماران سالمند
}

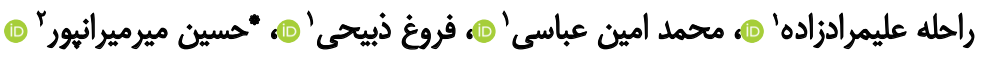 \\ 1. واحد توسعه تحقيقات بالينى، بيمارستان فيروزآبادى، دانشكاه علوميزشكى ايران، تهران، ايران.

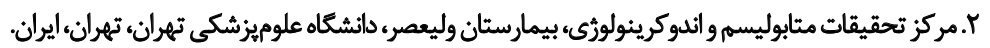

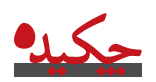

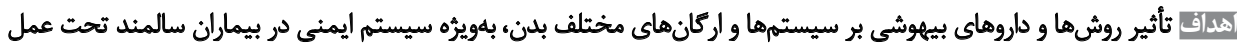

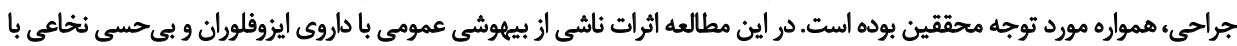

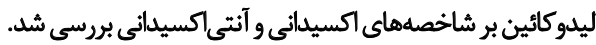

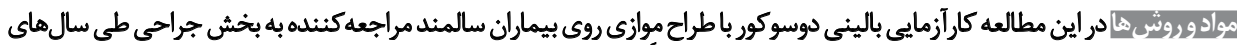

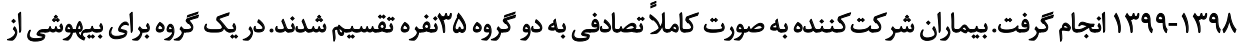

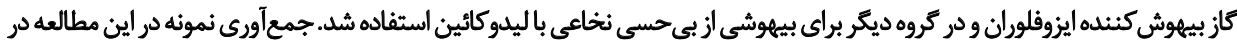

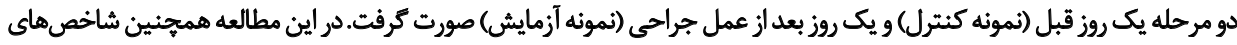

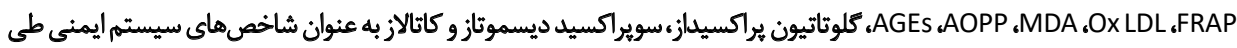

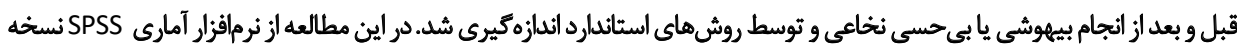

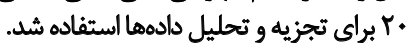

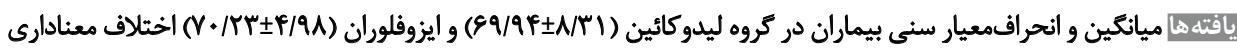

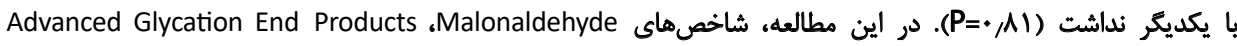
Superoxide Dismutase oxidized LDL Ferric Reducing Ability of Plasma ،Catalase ،Glutathione Peroxidase

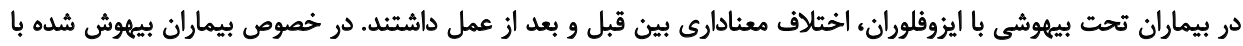

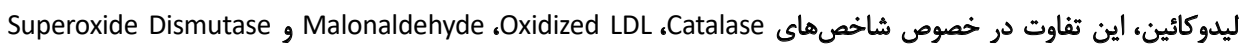

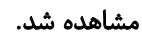

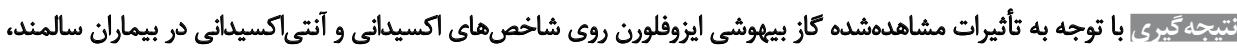

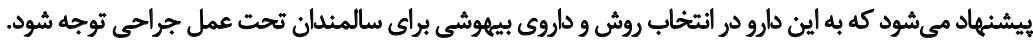

\author{
تاريخ دريافت: 10 تير

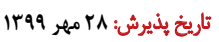

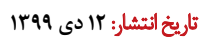

عمل جراحى از اهميت خاصى برخوردار است [ r [ ].

dotô

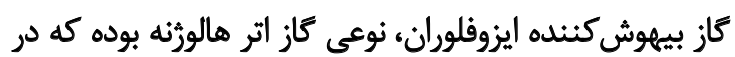

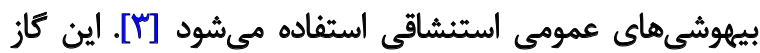

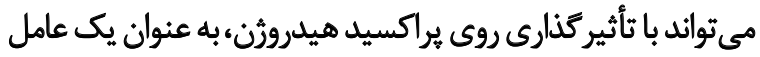

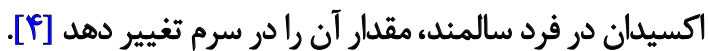

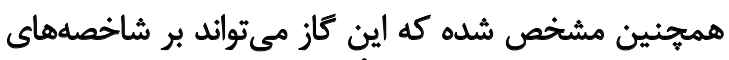

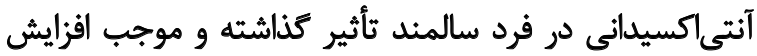

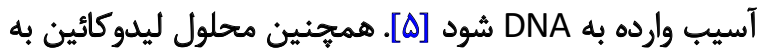

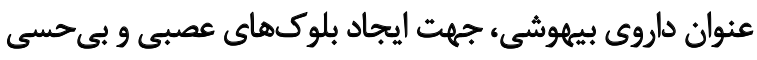
تأثير روشها و داروهاى بيهوشى بر سيستمها و اركانهاى

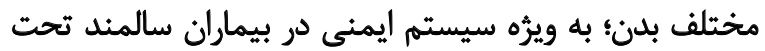

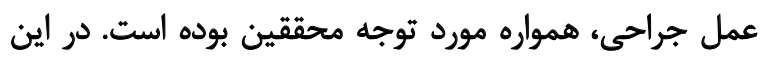

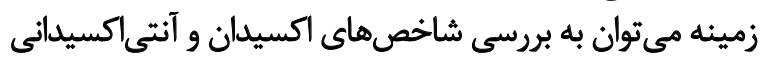

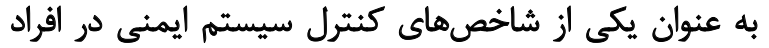

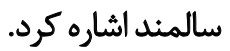

اين شاخص ها ارتباط اساسى باسيستم ايمنى بدن فرد سالمند

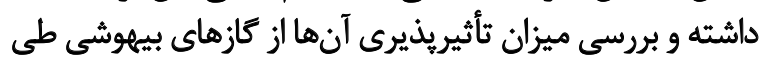
ㄷ․ 
بيهوشى عمومى با داروى ايزوفلوران و بيى بحسى نخاعى با باني

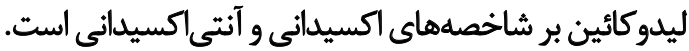

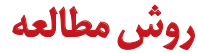

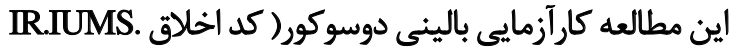
شركت

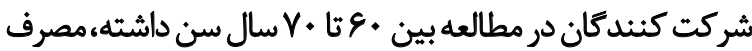

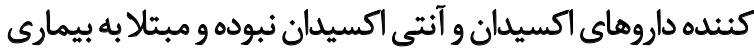

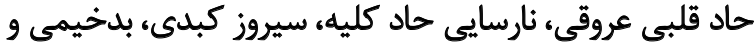

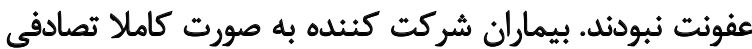

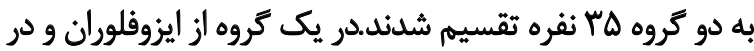
كروه ديكر از بى حسى نخاعى با ليدوكائين استفاده كرديد.

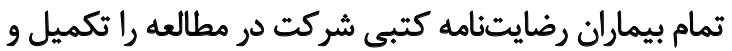

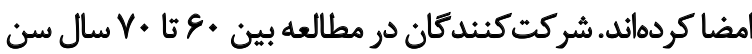

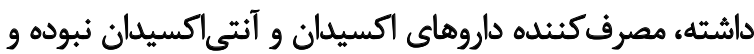

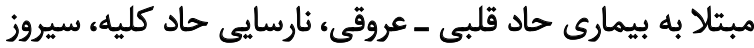
كبدى، بدخيمى و عفونت فعال نيز نبودندي

طى انجام مطالعه، آحر شركت كنيدكان از ادامه مطالعه منصرف

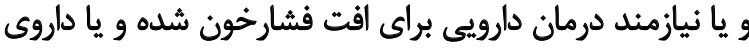
كورتيكواستروئيد دريافت كردند، از مطالعه خارج شدارند در اين مطالعه بيماران شركت شكننده به صورت كاملاً تصادفى

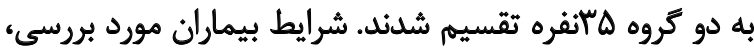

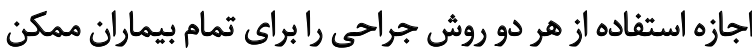

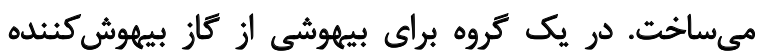

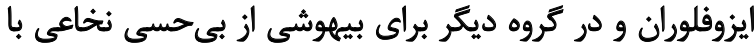
ليدوكائين استفاده شد.

متغيرهاى تنسنجى بيماران شامل سن، جنس، وزن، شاخص

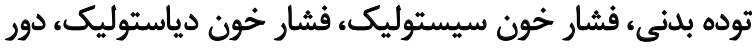

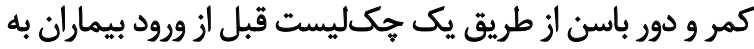

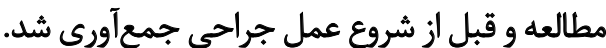

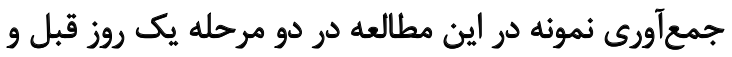

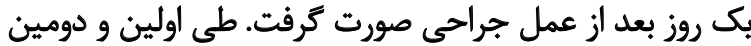

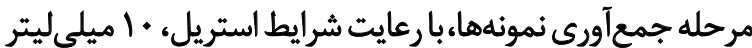

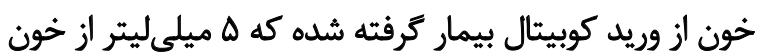

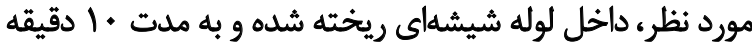

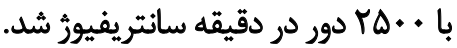
سيس T/ه ميلى ليتر سرم از ميزان خون اخير، جدا شده و

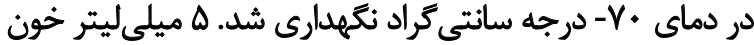

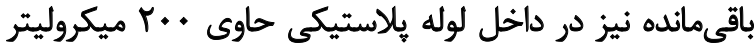

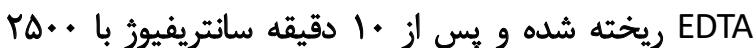

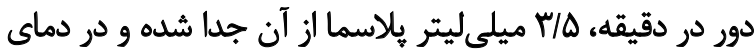

نخاعى استفاده مىشود [\&].

بي حسى نخاعى با ليدوكائين نسبت به بيهوشى عمومى،

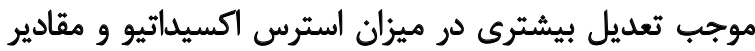

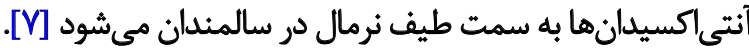

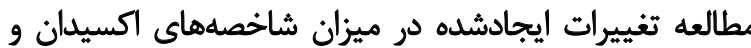

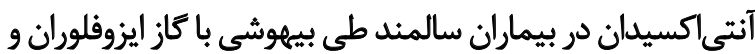

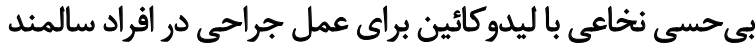

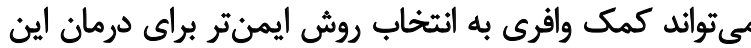
كروهاز بيماران مبتلا به بيمارى زميئلهاي كند.

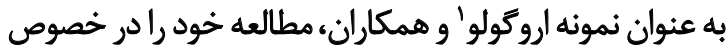

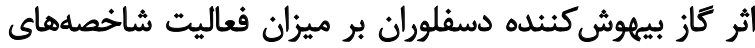

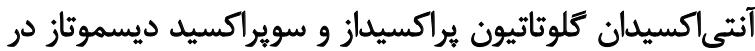

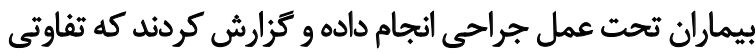

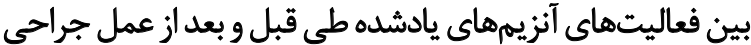

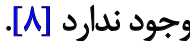

با وجود اينعسيلان ' و همكاران در مطالعه خود كَّزارش كردند

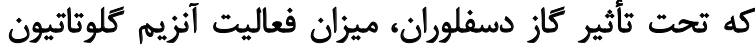

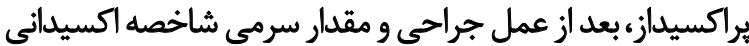

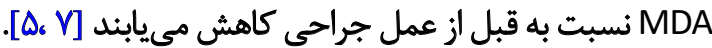

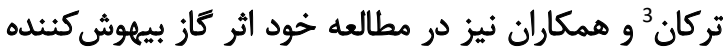

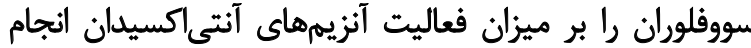

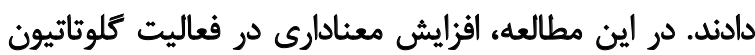

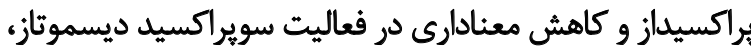

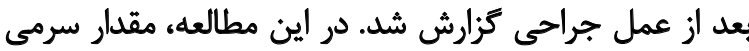

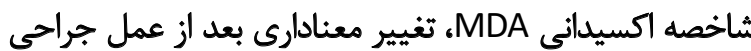

نسبت به قبل از عمل نداشت [9].

با وجود اين، در مطالعه سيواجى" و همكاران، مقدار سرميى دئي

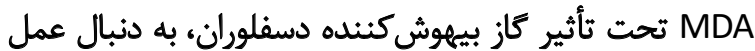

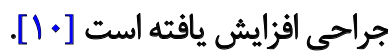

در مطالعات فوق، هرجند توجه وافرى به تحقيق در خصوص

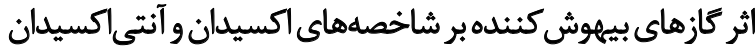

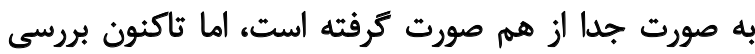

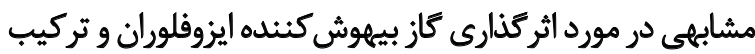

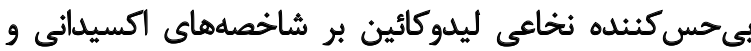

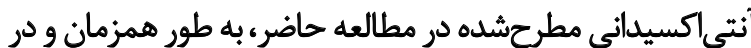
يك مجموعه مطالعاتى مشترك انجام نشده است.

هدف از انجام اين مطالعه، بررسى و مقايسه اثرات ناشى از 
جدول ا. توزيع فروانى متغيرهاى جمعيتشُناختى بين شركت كنئدكان در مطالعه

\begin{tabular}{|c|c|c|c|}
\hline $\mathbf{P}$ & كروه ايزوفلوزان & كورو ليدوكائين & متغير هاى مورد مطالعه \\
\hline \multirow{2}{*}{.199} & iv & IV & مرد ل \\
\hline & w & M & زن \\
\hline$\cdot|A|$ & $V \cdot / \pi \pm T / q u$ & Eq/qTINYI & سن \\
\hline $.1 \Delta \Delta$ & $\mid E T / N \pm N T Y$ & $\mid E T / M \pm \Delta / 1 \Delta$ & ق ق قد \\
\hline.$/ \mu$ & VV/IEIT/Tr & $V \in / \pi \Psi \pm I V / r \pi$ & وزن \\
\hline - per & WOPINDI & $1 . . / \% 9 \pm 11 / 9$. & دور شكم \\
\hline
\end{tabular}

每

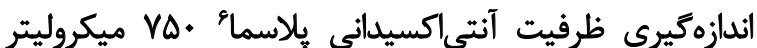

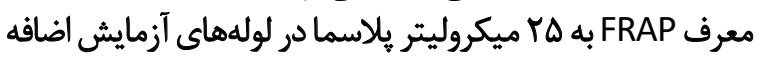

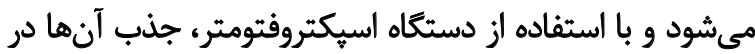
طول موج كوه قرائت شد.

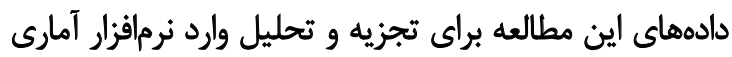

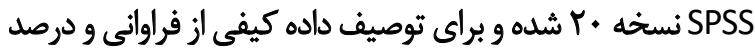

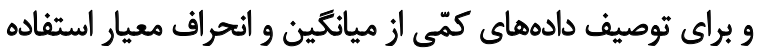

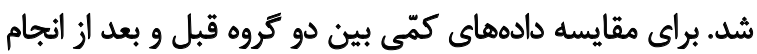

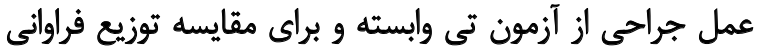

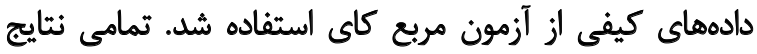
آزمونهاى آمارى كمتر از هـ ٪ إن معنادار تلقى شد.

يافتهاهيا

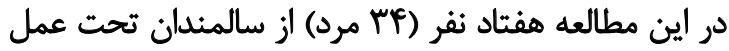

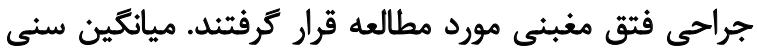

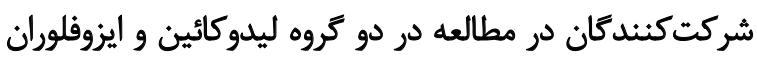

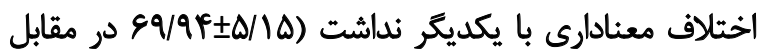

6. Ferric Reducing Ability of Plasma (FRAP)
منهاى •V درجه سانتى كراد نكهدارى شد. نمونهماي به دست

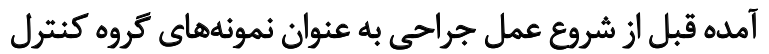

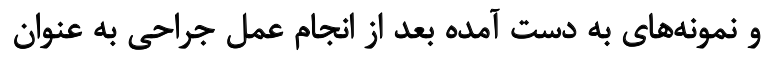
نمونههاى گروه آزمايش در نظر گرفته شدند.

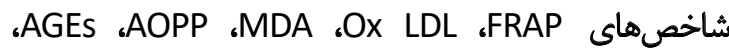

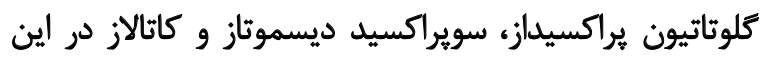

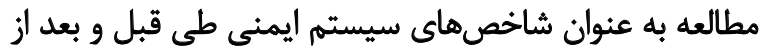

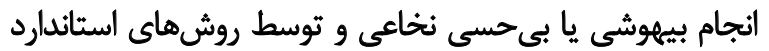
اندازهزيرى شد. از رنغسنجى آنزيمى براى اندازمكيرى ميزان فعاليت

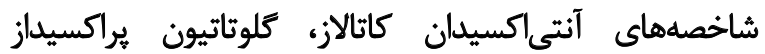

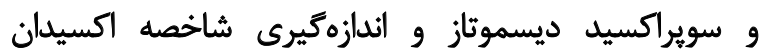
استفاده شده و اندازمكيرى شاخصه

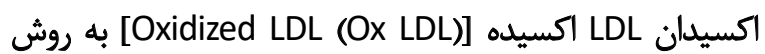

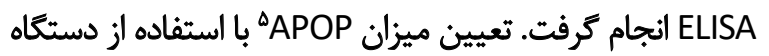

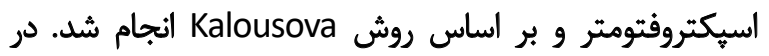

5. Advanced Oxidation Protein Products

جدول r. توزيع فراوانى شاخص اكسيدان و آنتىاكسيدانى قبل و بعد از عمل جراحى در بيماران كروه ايزوفلوران

\begin{tabular}{|c|c|c|c|}
\hline \multicolumn{3}{|c|}{ ميانكين||نحراف معيار } & \multirow{2}{*}{ هتغيرهاي مورد مطاله } \\
\hline $\mathbf{P}$ & يس از عمل & بيش از عمل & \\
\hline$<+1 \cdot+1$ & er/lrta/rr & $\varepsilon \& / 9 \vee \pm N / N$ & Advanced Glycation End Products \\
\hline.$/ \mu f$ & IFV/AVETN+1 & $\mid A T / F+ \pm T \Delta / T q$ & Advanced Oxidation Protein Products \\
\hline$<\bullet / .+1$ & $r / Q T \pm \cdot / \Delta F$ & $r / Q u \pm \cdot / \Delta V$ & Malonaldehyde \\
\hline$<\cdot 1 \cdot .1$ & $\mid r / I F \pm V / A T$ & $1 W / \Delta \cdot \pm 1 / V \varepsilon$ & Oxidized LDL \\
\hline$<+1 \cdot+1$ & $1.12 / M E \pm 1 r g / \Delta f$ & 1.VW/RTIIYNAS & Ferric Reducing Ability of Plasma \\
\hline$<\cdot 1 \cdot+1$ & $r / r \cdot \pm \cdot / r \Delta$ & $1 / Q \& \pm+/ T$ & Catalase \\
\hline.$/ \cdot r$ & $M / H \pm N \cdot F$ & $A F / Y r \pm V / \Lambda$. & Glutathione Peroxidase \\
\hline $.1++1$ & $r / m g \pm / r i$ & $m / q \Delta \pm+/ 41$ & Superoxide Dismutase \\
\hline
\end{tabular}

每 
جدول با. توزيع فراوانى شاخص اكسيدان و آنتىاكسيدانى قبل و بعد از عمل جراحى در بيماران كروه ليدوكائين

\begin{tabular}{|c|c|c|c|}
\hline \multicolumn{3}{|c|}{ ميانغين+|نحراف معيار } & \multirow{2}{*}{ متغير هاى مورد مطالعه } \\
\hline $\mathbf{P}$ & 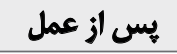 & ييش از عمل & \\
\hline.$/ 18$ & $9 / 99 \pm 7 / 91$ & $8 \Delta / \Delta V \pm N A \Delta$ & Advanced Glycation End Products \\
\hline .1 .94 & $|Q| / r q \pm \mid q / A V$ & $\mid \Delta F / R A \pm I F / V A$ & Advanced Oxidation Protein Products \\
\hline$<\cdot 1 . .1$ & $m / \cdot \pm \cdot / m$ & $r / T r \pm . / R q$ & Malonaldehyde \\
\hline$<+1 \cdot+1$ & $|V| \cdot \mid \pm \cdot / q r$ & IV/ret./ar & Oxidized LDL \\
\hline $1 \cdot \mathrm{N}^{4}$ & $M Q / N \pm E q / W$ & 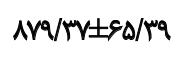 & Ferric Reducing Ability of Plasma \\
\hline$<+1++1$ & $r / r g \pm+/ r r$ & $r /|q \pm+/ r|$ & Catalase \\
\hline.$M I Y$ & $\Delta \Delta / A \vee \pm \Delta / V \Delta$ & $A r / A r \pm 8 / 9 r$ & $\begin{array}{l}\text { Glutathione } \\
\text { Peroxidase }\end{array}$ \\
\hline$<+1 *+1$ & $m / q P \pm \cdot / p i$ & $r / / \Delta \pm . / M A$ & Superoxide Dismutase \\
\hline
\end{tabular}

وضعيت قبل و بعد از جراحى در بيهوشى نخاعى با ليدوكائين در

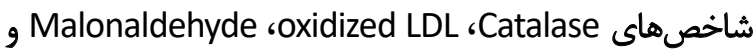
Superoxide Dismutase در جدول شماره ب درج شده است.

بحث

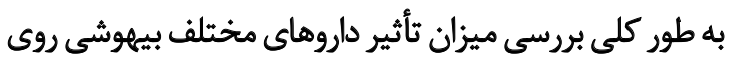

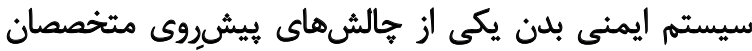

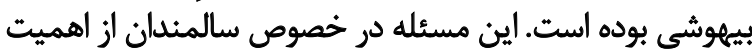
بيشترى نيز برخوردار است.

به عبارت ديخر، مطالعات مختلف به دنبال بررسى شاخصهاى

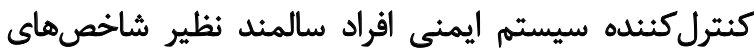

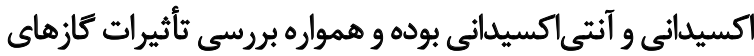

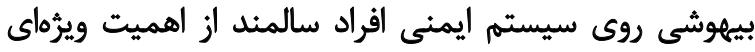

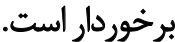

مشابه نتايج بهدستآمده در اين مطالعه، كاز بيهوش كنينده

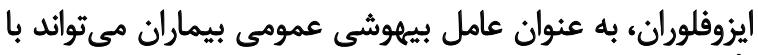

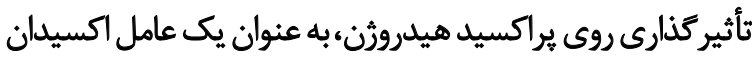

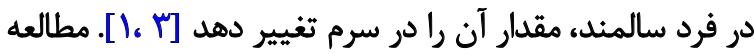

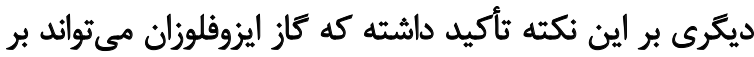

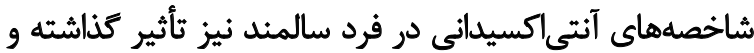

موجب افزايش آسيب وارده به DNA شود [ع] فيكان. از سوى ديكر ليدوكائين نيز در برخي از مطالعات براي ايجاد

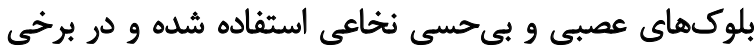

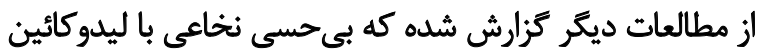

نيز

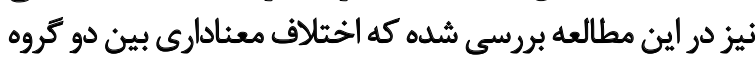

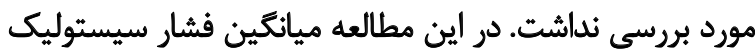

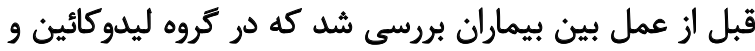

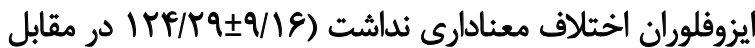

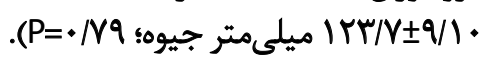

به همين شكل فشار دياستوليك نيز قبل از عمل اختلاف

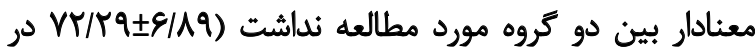

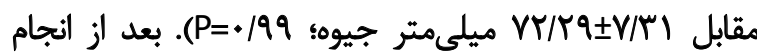

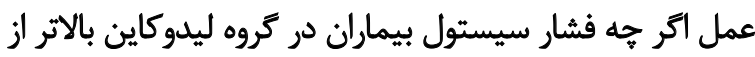

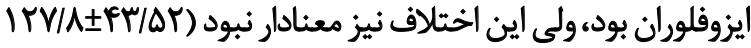

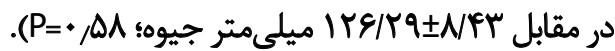
همجينين الكَوى مشابهى نيز در مقايسه ميزان فشار دياستوليك

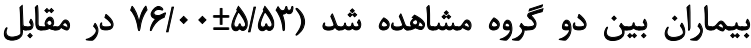

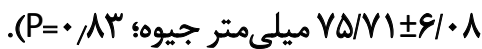

در بررسى و تجزيه و تحليل آمارى شاخصهاى اكسيدانى و

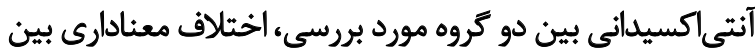

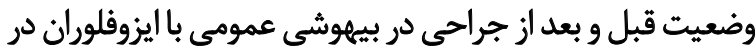

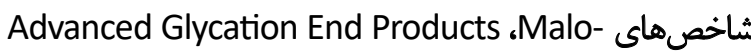
naldehyde ،oxidized LDL ،Ferric Reducing Ability of Plasma ،Catalase ،Glutathione Peroxidase و Superجدide Dismutase مشاهده شد. جزئيات اين مقايسهها در جدول شماره r درج شده است.

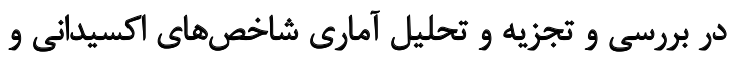

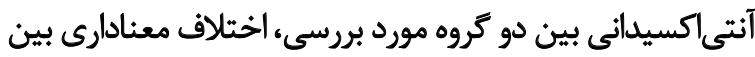




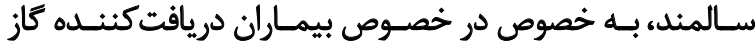

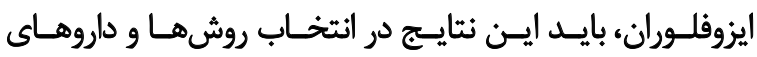

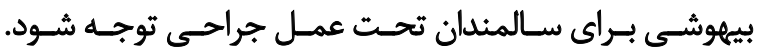

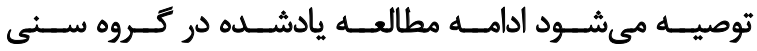

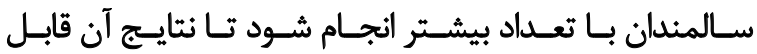

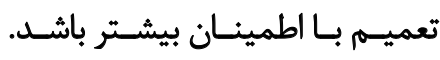

\section{يبيروى أز اصول الخاقاق يُوهش}

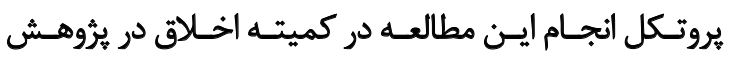

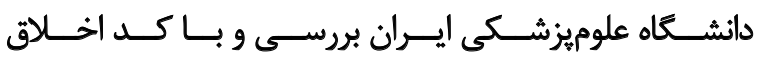

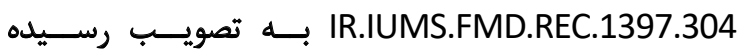

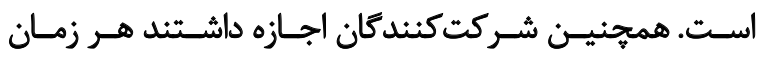

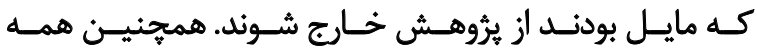

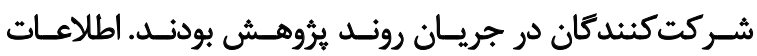

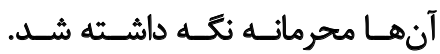

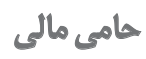

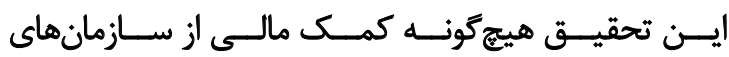

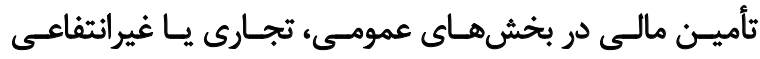
دريافت نكـرد.

$$
\text { مشاركت نويسئد مكان }
$$

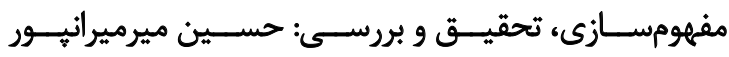

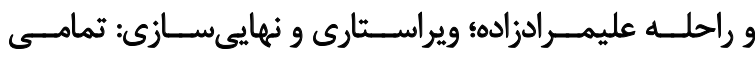

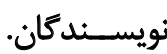

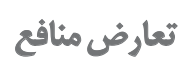

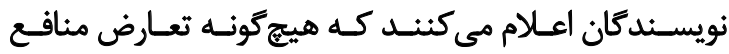

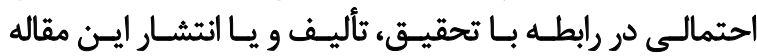

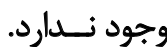

نسبت به بيهوشى عمومى، موجب تعديل بيشترى در ميزان

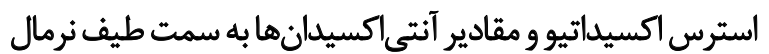
در سالمندان ميشود [F. V.

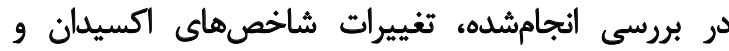

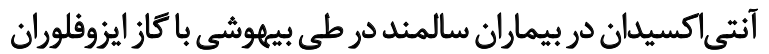

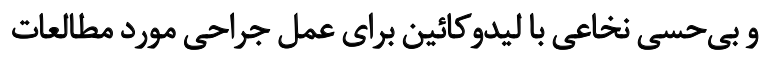

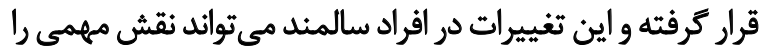

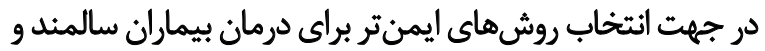
مبتلا به بيمارى زميئه اي ايفا كند.

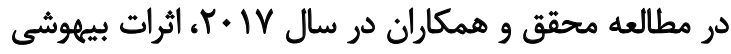

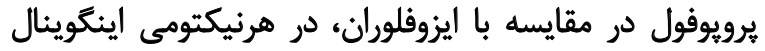

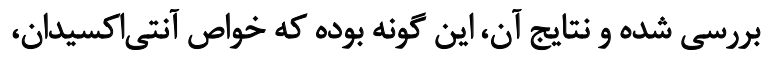

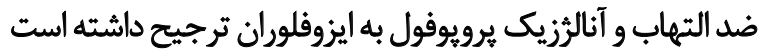

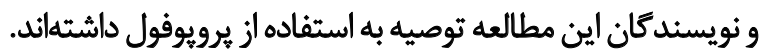
از آنجا كه فرايند جراحى هميشه همراه با استرس اكسيداتيو

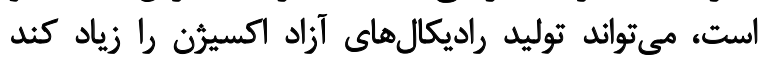

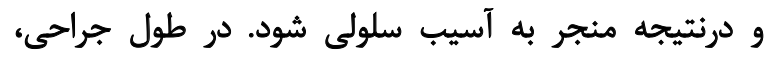
نوتروفيل هاى فعال شده مى توانئد مولكول اكسيرن رائ را به راديكال

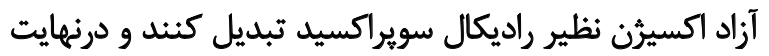

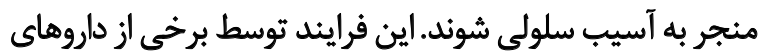

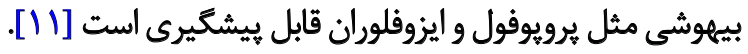

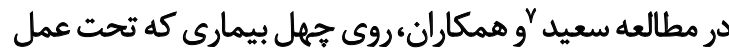

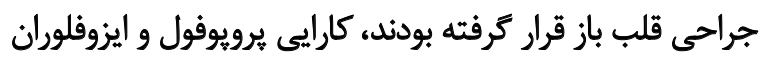

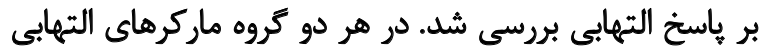

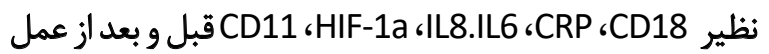

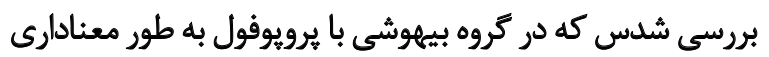

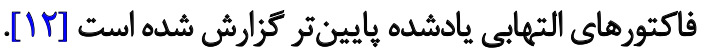
در اين مطالعه هم كه بر اساس اطلاعات ما براى اولين بار در

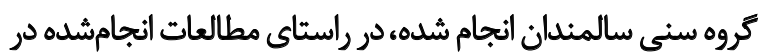
اين زمينه، شاخصهاى Advanced Glycation End Products ،Malonaldehyde ،Oxidized LDL ،Ferric Reducing AbilSu- $g$ ity of Plasma ،Catalase ،Glutathione Peroxidase peroxide Dismutase

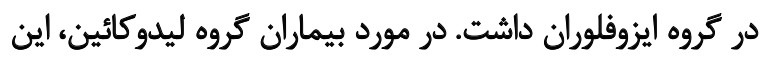
Malonaldehyde، Oxidized تفاوت در خصوص اين شانين Superoxide Dismutase و LDL، Catalase

\section{نتيجهيَيرى نهاييى}

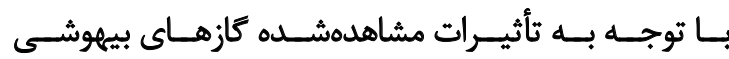

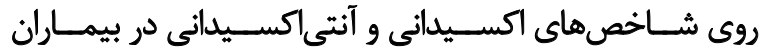




\section{References}

[1] Shafer SL. The pharmacology of anesthetic drugs in elderly patients. Anesthesiology Clinics of North America. 2000; 18(1):1-29. [DOI:10.1016/S0889-8537(05)70146-2]

[2] Peacock JE, Lewis RP, Reilly CS, Nimmo WS. Effect of different rates of infusion of propofol for induction of anaesthesia in elderly patients. British Journal of Anaesthesia. 1990; 65(3):346-52. [DOI:10.1093/bja/65.3.346] [PMID]

[3] Niedermeyer E, Silva FHLd. Electroencephalography: Basic Principles, Clinical Applications, and Related Fields. Philadelphia: Lippincott Williams \& Wilkins; 2005. https://books. google.com/books/about/Electroencephalography. html?id=tndqYGPHQdEC

[4] Pharmacists. TASoH-S. Lidocaine Hydrochloride (Antiarrhythmic). The American Society of Health-System Pharmacists; 2015.

[5] Ceylan BG, Nazıroğlu M, Uğuz AC, Barak C, Erdem B, Yavuz L. Effects of vitamin $C$ and $E$ combination on element and oxidative stress levels in the blood of operative patients under desflurane anesthesia. Biological Trace Element Research. 2011; 141(1-3): 1625. [DOI:10.1007/s12011-010-8712-3] [PMID]

[6] Wilson WC, Swetland JF, Benumof JL, Laborde P, Taylor R. General anesthesia and exhaled breath hydrogen peroxide. Anesthesiology. 1992; 76(5):703-10. [DOI:10.1097/00000542-19920500000007] [PMID]

[7] Ceylan BG, Yilmaz F, Eroglu F, Yavuz L, Gulmen S, Vural H. Oxidant and antioxidant activities of different anesthetic techniques. Saudi Medical Journal. 2009; 30(3):371-6. [PMID]

[8] Eroglu F, Yavuz L, Ceylan BG, Yulmaz F, Eroglu E, Delibas N, et al. New volatile anesthetic, desflurane, reduces vitamin $E$ level in blood of operative patients via oxidative stress. Cell Biochemistry and Function. 2010; 28(3):211-6. [DOI:10.1002/cbf.1641] [PMID]

[9] Kulacoglu H, Ozdogan M, Gurer A, Ersoy E, Onder AD, Duygulu SD, et al. Prospective comparison of local, spinal, and general types of anaesthesia regarding oxidative stress following Lichtenstein hernia repair. Bratislavske Lekarske Listy. 2007;108(8):335-9. [PMID]

[10] Sivaci R, Kahraman A, Serteser M, Sahin DA, Dilek ON. Cytotoxic effects of volatile anesthetics with free radicals undergoing laparoscopic surgery. Clinical Biochemistry. 2006; 39(3):293-8. [DOI:10.1016/j.clinbiochem.2006.01.001] [PMID]

[11] Mohaghegh T, Yazdi B, Norouzi A, Fateh Sh, Modir H, Abolfazl Mohammadbeigi A. Effect of intravenous anesthesia with propofol versus isoflurane inhalation anesthesia in postoperative pain of inguinal herniotomy: A randomized clinical trial. Medical Gas Research. 2017; 7(2):86-92. [DOI:10.4103/2045-9912.208511] [PMID] [PMCID]

[12] Sayed S, Idriss NK, Sayyedf HG, Ashry AA, Rafatt DM, Mohamed AO, et al. Effects of propofol and isoflurane on haemodynamics and the inflammatory response in cardiopulmonary bypass surgery. British Journal of Biomedical Science. 2015; 72(3):93-101. [DOI:10.1080/09674845.2015.11666803] [PMID] 
This Page Intentionally Left Blank 